\title{
Intestinal infarction caused by carcinoid associated elastic vascular sclerosis: early presentation of a small ileal carcinoid tumour
}

\author{
J N HARVEY, M E DENYER, AND P DACOSTA \\ From the Departments of Medicine and Pathology, Seacroft Hospital, Leeds
}

SUMmaRY We describe a patient with extensive ischaemic necrosis of the ileum as a result of elastic vascular sclerosis (EVS). A $2 \mathrm{~cm}$ carcinoid tumour was located nearby with microscopic evidence of spread to regional lymph nodes. Severe intestinal ischaemia caused by carcinoid associated EVS may be the presenting feature of small carcinoid tumours resulting in their early diagnosis.

Carcinoid tumours which have metastasised from GI tract to liver may present with the well known carcinoid syndrome, by which time definitive treatment is usually impossible. Surveys suggest that this syndrome is found in approximately $5 \%$ of carcinoid tumours, ${ }^{12}$ the remainder being found incidentally or presenting with symptoms referable to their site of origin. Such symptoms may be the result of small bowel obstruction (partial or complete), intussusception or bleeding. The present report describes an alternative manifestation: intestinal gangrene caused by elastic vascular sclerosis (EVS). In contrast with previous reports the present case indicates that severe intestinal ischaemia may be the result of EVS in association with a small carcinoid tumour without macroscopic spread.

\section{Case report}

An 85 year old woman was admitted with a one month history of intermittent nausea and vomiting after meals. These symptoms had worsened two days immediately before admission and she had developed abdominal pain, initially in the lower abdomen but subsequently generalised. Her appetite had been poor with weight loss of an indeterminate amount, probably slight. Her bowel habit had been

Address for correspondence: Dr J N Harvey, Department of Medicine, G Floor, Martin Wing, General Infirmary, Leeds LS1 3EX.

Accepted for publication 21 October 1988. irregular. She had suffered with mild intermittent dysphagia over the previous two years, but denied dyspepsia or flushing attacks. She had had rheumatic fever when young but never any known ischaemic vascular disease. Prescribed therapy at admission was: digoxin, frusemide, amiloride, and motilium.

On examination she was mildly dehydrated and pyrexial: $37 \cdot 5^{\circ} \mathrm{C}$. Pulse rate was $96 \mathrm{bpm}$, atrial fibrillation, with a tapping apex and mitral diastolic murmer. Tenderness was maximal in the lower abdomen. Investigations showed a neutrophil leucocytosis $\left(11.8 \times 10^{9} / 1\right)$ but normal haemoglobin concentration $(13.5 \mathrm{~g} / \mathrm{dl})$. Plasma urea $11.8 \mathrm{mmol} / \mathrm{l}$, creatinine $141 \mathrm{umol} / /$, electrolytes and liver enzymes were normal. Blood cultures were negative, plasma digoxin undetectable, amylase 156 somogyi units. An echocardiogram showed mild to moderate mitral stenosis and mild aortic stenosis but no vegetations. There was left ventricular hypertrophy with good LV function. Abdominal ultrasound showed gall stones. Upper GI endoscopy revealed a hiatus hernia and oesophagitis, with bile reflux into the stomach. Plain abdominal radiographs showed slight distension of the small bowel but insufficient to be diagnostic of obstruction. A barium enema demonstrated extensive diverticular disease in the sigmoid and descending colon.

TREATMENT AND PROGRESS

Initially she was thought to have acute diverticulitis 
and oesophagitis. She was treated with ampicillin; gentamicin, metronidazole, cimetidine, and metoclopramide. Digoxin and diuretics were continued. Anticoagulants were withheld because of the risk of GI bleeding and because of the possible need for surgery. Her symptoms did not improve, and there was a persistent mild pyrexia up to $37.5^{\circ} \mathrm{C}$ and leucocytosis rising to $18.7 \times 10^{9} /$. The haemoglobin concentration fell slightly to $10.9 \mathrm{~g} / \mathrm{dl}$. The abdomen became distended with rebound tenderness. Repeat radiographs suggested small bowel obstruction. At laparotomy, ischaemic and necrotic ileum was resected and a limited right hemicolectomy performed. Initial postoperative progress was satisfactory but then her general condition deteriorated and she died on the eighth postoperative day.

\section{HISTOPATHOLOGICAL EXAMINATION}

The surgical resection specimen consisted of caecum and $60 \mathrm{~cm}$ ileum exhibiting extensive ischaemic necrosis with evidence of peritonitis. Midway along it the lumen was slightly constricted by an ulcerated tumour, $2 \mathrm{~cm}$ in maximum diameter, which appeared to invade and just penetrate the wall. Histology showed this to be a malignant carcinoid tumour, which exhibited a positive argentaffin and argyrophilic reaction. Immunohistochemistry showed the presence of serotonin but was negative for gastrin, glucagon, insulin, somatostatin, and ACTH. The ileal arteries showed a curious thickening of their walls, with near obliteration of the lumens, most obvious in the larger arteries of the vascular pedicle, although lesser changes were seen in smaller arteries and some veins near the tumour. This was caused by a dramatic endarterial proliferation of elastic tissue within the limits of the internal elastic lamina, together with elaboration of elastic tissue within the adventitia forming a mantle around the vessels (Figure). The small vessels within and immediately adjacent to the tumour were virtually normal. A few tumour cells were found in the regional lymph nodes on microscopic examination.

At necropsy there was no evidence of residual carcinoid tumour, or of carcinoid heart disease, and death was attributed to congestive cardiac failure as a result of severe ischaemic heart disease and old rheumatic mitral valve disease. Many ileal arteries up to $20 \mathrm{~cm}$ from the resection site showed evidence of elastic vascular sclerosis, but other systemic arteries were unremarkable.

\section{Discussion}

This elderly patient presented difficulties in management. The clinical findings at admission were not specific for small bowel disease, and the presence of other pathology: oesophagitis and widespread colonic diverticula resulted in delay in diagnosing the main cause of her symptoms. The carcinoid tumour subsequently found was small and, in the absence of liver metastases would be unlikely to give rise to symptoms suggestive of the carcinoid syndrome.

Elastic vascular sclerosis is an uncommon cause of small bowel ischaemia, and frank infarction is rare. This pathological entity was first described by Anthony and Drury, ${ }^{3}$ who reviewed 100 carcinoid tumours. They found an apparent increase in vascular elastic tissue in 17 of 25 specimens containing ileal carcinoid tumours, but in none of $\mathbf{7 5}$ appendicular, rectal or other carcinoids. This resulted in ischaemic ileal necrosis in only four. In an earlier series of carcinoid tumours from the Mayo clinic, Moertel et $a l^{2}$ reported ischaemic necrosis in only four of 209 specimens. Vascular elastosis has been described in association with a jejunal carcinoid ${ }^{4}$ but not with carcinoids of hindgut origin. In all cases with elastosis the tumour had invasive characteristics, and in this respect the tumour described here appears typical. In a review of 26 reported cases with intestinal infarction, Eckhauser et al $^{5}$ were able to categorise spread of the disease in 20. All had extensive metastases to regional nodes, and $12(60 \%)$ had remote disease. Similarly substantial metastatic spread was noted in patients with intestinal infarction by Palvio et al (one case), ${ }^{6}$ Qizilbash (two), ${ }^{4}$ Warner (two), ${ }^{7}$ and Chapuis (one) ${ }^{8}$ The present case contrasts with this and shows that a small tumour with only microscopic spread to local lymph nodes can cause severe EVS with intestinal infarction.

In their original description, Anthony and Drury ${ }^{3}$ suggested that EVS is a local response to the elaboration of an active substance by the tumour. Because both arteries and veins (to a lesser extent) are affected, and the vessels within or near the tumour appear to be spared, a direct or downstream effect seems unlikely. Possibly an active substance passes into the mesenteric lymphatics and subsequently diffuses out. Hence, only tumours which invade the mesentery exhibit vascular elastic changes. Alternatively, Qizilbash ${ }^{4}$ suggests the elastosis is a direct effect of the tumour on the fibroblast or smooth muscle cell in the vascular wall. Our own case, however, illustrates sparing of vessels within and immediately adjacent to the tumour, and is therefore more consistent with the former explanation.

Carcinoid tumours are well known to produce changes in the endocardium, valves and great vessels. These lesions are caused by collagenous fibrosis, occurring within the intima and containing little elastic tissue. ${ }^{9}$ Fibrosis also accounts for the well 


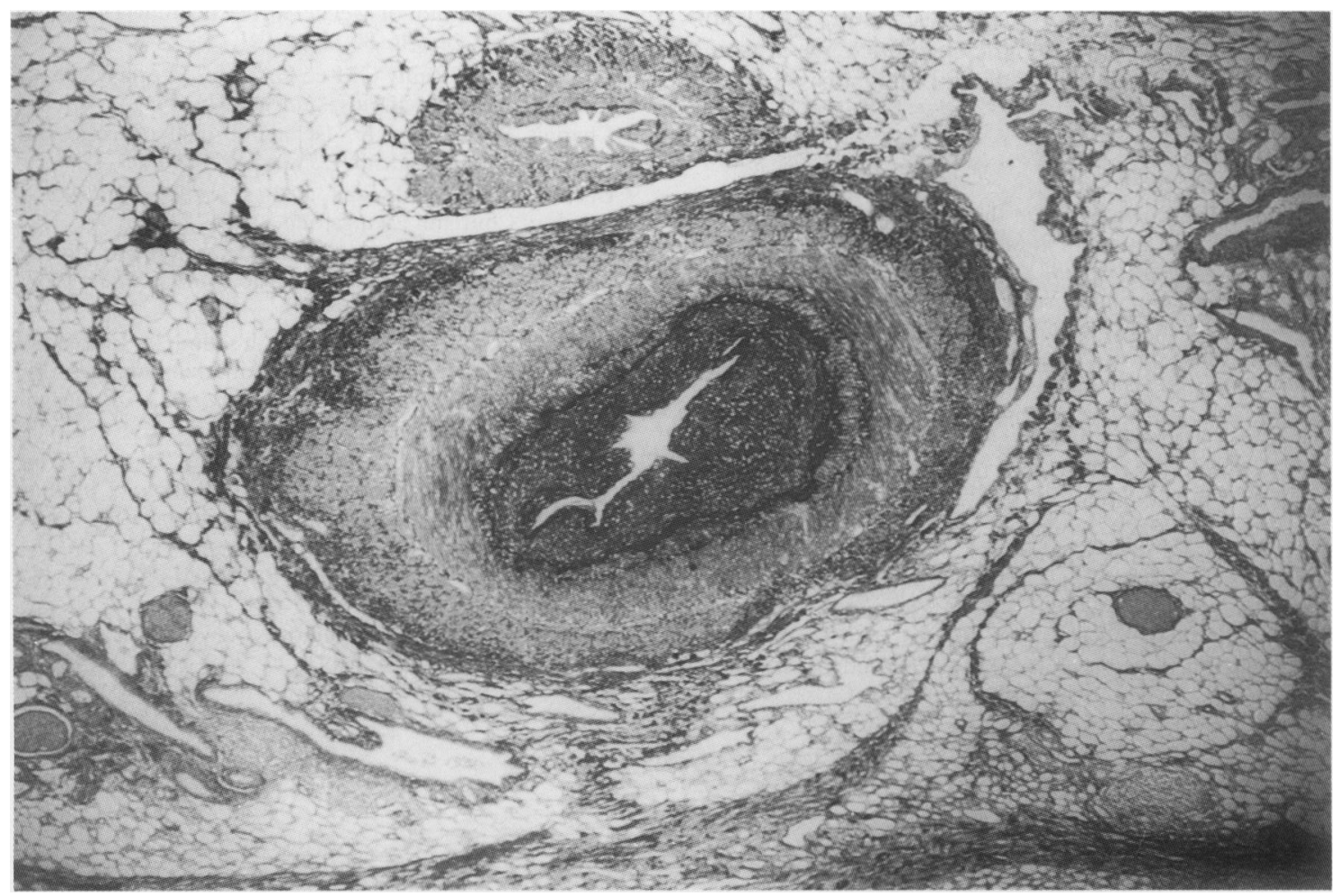

Figure Medium sized muscular artery showing conspicuous elastic sclerosis of the intima, with a lesser degree of adventitial elastosis (Miller's elastic and Van Gieson stain).

documented changes in the mesentery, which may cause small bowel obstruction by tethering and kinking. ${ }^{2}$ The histology of the ileal vascular lesions is distinct in that they contain predominantly elastic tissue, laid down in the intima and the adventitia of vessels. The intimal elastosis has a dense obliterative quality unlike the concentric lamellar fibroelastic intimal hyperplasia seen in hypertensive arteriopathy, and a unique feature is the formation of an adventitial mantle of elastic tissue.

Serotonin is thought to be responsible for the cardiac valvular fibrosis found in the carcinoid syndrome: the demonstration of identical lesions in patients treated with methysergide, a drug which can act as a partial agonist at tryptaminergic receptors, supports this contention. ${ }^{11}$ The agent responsible, however, for the changes in mesenteric vascular elastic tissue is unknown. Carcinoid tumours are capable of synthesising a variety of amines and peptides. In addition to serotonin, enterochromaffin cell tumours are capable of producing 5hydroxytryptophan, ACTH, substance $\mathrm{P}$, motilin, met-enkephalin, $\beta$-endorphin, neurotensin, gastrin, and somatostatin. ${ }^{112}$ Several of these have actions on smooth muscle - for example, substance $P$, motilin, and neurotensin,,$^{13}$ and might contribute to the development of vascular elastosis. Midgut carcinoids are recognised to be more endocrinologically active than those arising from hindgut, ${ }^{12}$ which might explain the fact that elastic vascular sclerosis has not been found in the latter.

Carcinoid tumours are characteristically slow growing and have a good prognosis if discovered early before metastasis. Prognosis is related to site: those in the vermiform appendix having a five year survival rate of $99 \%$ compared with $54 \%$ for those of small intestinal origin. ${ }^{14}$ The poor outcome of the latter is likely to be the result of the later development of symptoms, which most commonly are nonspecific, consisting of abdominal pain and diarrhoca. In Moertel's series, ${ }^{2}$ most (95\%) patients with a symptomatic carcinoid had confirmed metastases. The few reported cases of carcinoid associated intestinal infarction have generally been associated with distant metastases, or substantial nodal deposits thought in some patients to cause vascular obstruction. ${ }^{2}$ Significant ischaemia caused by elastic vascular sclerosis may result in earlier presentation. The 
present case illustrates that such ischaemia with frank infarction can indicate the presence of a small carcinoid tumour without distant metastasis.

\section{References}

1 Linell F, Mansson K. On the prevalence and incidence of carcinoid tumours in Malmo. Acta Med Scand 1966; 179 [suppl 444]: 377-82.

2 Moertel CG, Sauer WG, Dockerty MB, Baggenstoss AH. Life history of the carcinoid tumour of the small intestine. Cancer 1961; 14: 901-12.

3 Anthony PP, Drury RAB. Elastic vascular sclerosis of mesenteric blood vessels in argentaffin carcinoma. J Clin Pathol 1970; 23: 110-8.

4 Quizilbash AH. Carcinoid tumours, vascular elastosis, and ischemic disease of the small intestine. Dis Colon Rectum 1977; 20: 554-60.

5 Eckhauser FE, Argenta LC, Stroedel WE, et al. Mesenteric angiopathy, intestinal gangrene, and midgut carcinoids. Surgery 1981; 90: 720-8.

6 Palvio DHB, Kristensen ES, Falk E. Intestinal ischaemia due to vascular elastosis caused by metastasiz- ing carcinoid tumor of Meckel's diverticulum. Dis Colon Rectum 1985; 28: 746-8.

7 Warner TF, O'Reilly G, Lee GA. Mesenteric occlusive lesion and ileal carcinoids. Cancer 1979; 44: 758-62.

8 Chapuis $P$, Weedon $D$. Ischaemic ileal necrosis and carcinoid tumour. Aust NZ J Surg 1976; 46: 63-4.

9 Becker AE, Anderson RH. Cardiac pathology. London: Churchill Livingstone, 1983; 423.

10 Graham JR. Cardiac and pulmonary fibrosis during methysergide therapy for headache. Am J Med Sci 1967; 254: 1-12.

11 Solcia E, Capella C, Buffa R, Usellini L, Fiocca R. Cytology and biology of gut endocrine tumours. In: Bloom SR, Polak JM. Gut hormones. Edinburgh: Churchill Livingstone, 1981; 542-6.

12 Lewin KJ, Ulich T, Yang K, Layfield L. The endocrine cells of the gastrointestinal tract. Pathology annual. Norwalk, Connecticut: Appleton-Century-Crofts. 1986; 21/2: 181-215.

13 Glass GJ. Gastrointestinal hormones. New York, Raven Press: 1980.

14 Godwin JD. Carcinoid tumours: an analysis of 2837 cases. Cancer 1975; 36: 560-9. 